\title{
Research on Laser Ranging Method based on CMOS Image Sensor
}

\author{
Quanzi Wang ${ }^{1,}$, , Zijian Yang ${ }^{2, b^{*}}$, Pan Wei ${ }^{3, c}$ \\ ${ }^{1}$ Jiangsu Key Laboratory of Large Engineering Equipment Detection and Control, Xuzhou Institute \\ of Technology, Xuzhou, Jiangsu Province, 221000,China \\ 2 Jiangsu Key Laboratory of Large Engineering Equipment Detection and Control, Xuzhou Institute \\ of Technology, Xuzhou, Jiangsu Province, 221000,China \\ ${ }^{3}$ Jiangsu Key Laboratory of Large Engineering Equipment Detection and Control, Xuzhou Institute \\ of Technology, Xuzhou, Jiangsu Province, 221000,China \\ a'wquanzi0219@163.com, byangzjpp@126.com, c $846948728 @ q q . c o m$
}

Key words: Triangle Ranging Method Regional Squared centroid Method CMOS Image Sensor

Abstract: A new regional squared centroid method is proposed to design a ranging System adopted MCU, CMOS image sensor and direct laser triangulation method, after analyzing the advantages and disadvantages of the laser triangulation method. And the principle, hardware settings and data processing of this system are also introduced in this paper.

\section{Introduction}

In the case of the rapid development of industrial technology, the traditional contact measurement technology has been unable to meet industrial requirements, and laser ranging technology, one of the important applications of laser, is widely used in modern industry, especially in the automotive industry and rapid manufacturing, for its non-contact measurement and accurate calculation results. Laser triangulation ranging technology because of its simple structure, convenient use, fast measurement speed and efficient real-time processing ability, is widely used in range, mold design, medical $3 \mathrm{~d}$ measurement, etc. However, to improve the precision of laser rangefinder is still a problem to be solved in the field of laser ranging.

\section{The structure and principle of ranging System}

\section{The principle of ranging}

According to the relationship between the incident ray and the surface normal of a measured object, optical triangulation method is divided into oblique and direct type, which, as shown in figure 1, mainly consists of a laser emitter, a lens, CMOS image sensor and so on. Based on the triangular relationship and Newton's formula, shown in Figure 1, I can obtain ${ }^{[1]}$ :

$$
\mathbf{X}=\mathrm{L}-\frac{b \cdot \Delta x \cdot \sqrt{L^{2}+l^{2}}}{a \cdot l \pm \Delta x \cdot L}
$$

When measured distance is greater than the reference distance, we need to take a minus sign; otherwise, we take a plus sign ${ }^{[2]}$.

$$
\mathbf{X}=\frac{\Delta \mathrm{x} \bullet\left(f \bullet \sqrt{L^{2}+l^{2}}-l^{2}\right)-f \bullet l \bullet L}{X \bullet L-f \bullet l}
$$


In the above two formulas, the letters "L" represents the reference distance corresponding to the center of the photosensitive of the CMOS image sensor, and "l" represents the baseline length, namely the distance from the laser emission port to the center of the imaging system. " $\Delta X$ " is the signed distance between the two image point of the object to be measured and the reference distance in the photosensitive surface. " $\mathrm{X}$ " is the measured distance and " $\mathrm{f}$ " is the focal length of the convex glass.

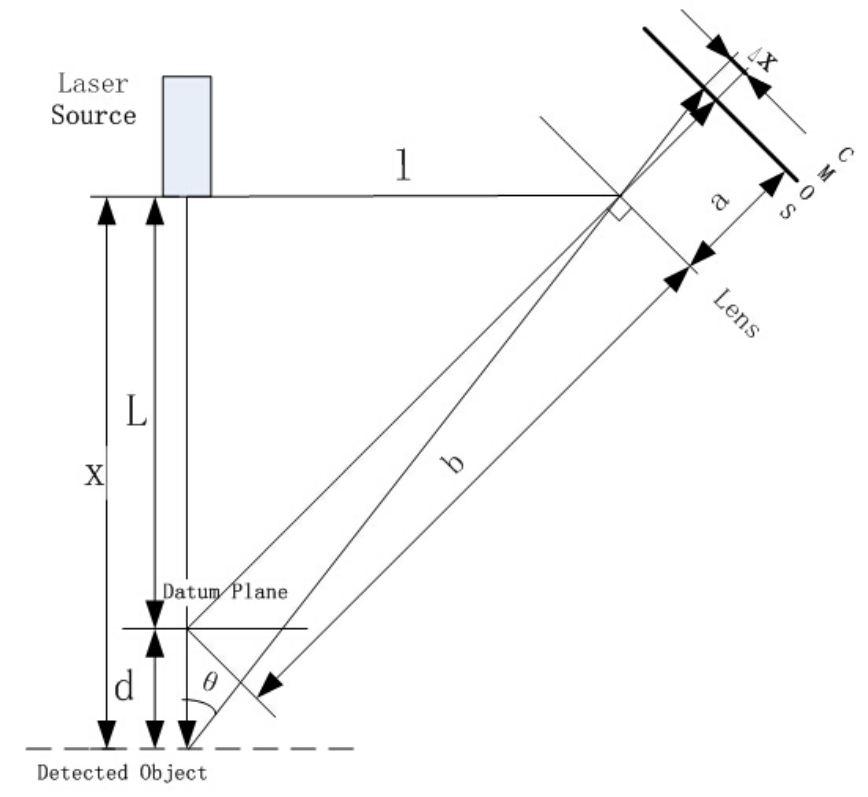

Figure 1

\section{Hardware design}

Freescale MC9S12XS128M is the master control chip for image acquisition, spot centroid solution and other data processing; the key parts of the system also include laser source with $5 \mathrm{mw}$, $650 \mathrm{~nm}$ red dot laser CMOS camera and 1602LCD for displaying distance. Via serial ports, we observe the effect of the image collected by MCU on the PC.

In this paper the calibration method is taking a measurement object, and every moving the object $150 \mathrm{~mm}$, recording the spot displacement on the CMOS. In order to reduce error, the average values of the sets of the recorded data are used as measurement value.

\section{Laser spot image processing}

\section{Image filtering}

At present, the commonly used filtering algorithms include mean filtering, median filtering, Gaussian filtering algorithm and these improved algorithms. And the median filter, whose main idea is to sort pixel according to the gray values and take the median gray value as the current value in one region, is a nonlinear filter.

The different gray values of background and its adjacent pixel is large through the nonlinear extension, and then replaces the gray values of noise by its adjacent pixels after sorting to overcome the blurred image details caused by average filtering and filter out noise while keeping image edges.

In this paper, the traditional median filtering is polished by retaining the value of the median and several nearby and replacing the pixel value with the average these values. In this way the polished algorithm has achieved good effect on eliminating the impact of noise ${ }^{[2]}$. 
The Typical methods to calculate spot centroid include the centroid method with threshold, squared cantroid method, curve fitting algorithm, etc. The image of the first method is just the superposition of the real images and kinds of noises so it is difficult to determine a reasonable threshold to extract the spot centroid ; Squared cantroid method adopts the square of gray level instead of gray level as weights and it shows that the points of larger gray levels near the center affect the central position, while reduce the proportion of self-noise and other system noises ${ }^{[3]}$. But the data processing speed is relatively slow.

This paper introduces regional squared centroid method to calculate the spot centroid. After the threshold, which is used to count the summation of gray levels which greater than this threshold in every line, is determined in one region, and then return the greatest summation line. Secondly, to read the line and find out the location of the highest gray level .Afterwards, a rectangle area, which is of fifty pixels near the position of the highest gray level, is made sure ${ }^{[2]}$.Finally, to take squared centroid method. In addition, the formulary of squared centroid method is expressed as:

$$
\bar{X}=\frac{\sum_{i=1}^{m} \mu_{\mathrm{i}}^{2} x_{i}}{\sum_{i=1}^{m} \mu_{i}^{2}} \text { (3) }
$$

In the above formulary $(1), \mathrm{X}=\left(\bar{X}_{-123}\right) \times 0.016 \mathrm{~mm}, \bar{X}$ is the coordinate of spot centroid .Consequently, we can get the distance with that formulary as long as the coordinate location of spot centroid is found out ${ }^{[1]}$. The regional squared centroid method is taken to further reduce the proportion of the self - noise influence on spot centroid position and improve the data processing speed, thereby reducing the error.

\section{Experimental data and error analysis}

The jitter of Y-axis value of the circle center, which is caused by some accidental factor, has no significant rules, and the value of Y-axis does not have an impact on the longitudinal measurement, so we only need to consider changes in the $\mathrm{X}$-axis ${ }^{[4]}$. In this paper, parameters are set, such as the focal length " $\mathrm{f} "=10 \mathrm{~mm}$, the baseline distance "l" $=80 \mathrm{~mm}$, and the baseline distance "L"=2000mm. And the experimental data is shown in table 1 :

Table 1 the experimental data

\begin{tabular}{|c|c|c|c|c|c|c|c|}
\hline $\mathrm{x} / \mathrm{pixel}$ & $\begin{array}{c}\text { Measured } \\
\text { Value } / \mathrm{mm}\end{array}$ & $\begin{array}{c}\text { Actual } \\
\text { Value/mm }\end{array}$ & Error/mm & $\mathrm{x} / \mathrm{pixel}$ & $\begin{array}{c}\text { Measured } \\
\text { Value } / \mathrm{mm}\end{array}$ & $\begin{array}{c}\text { Actual } \\
\text { Value } / \mathrm{mm}\end{array}$ & Error /mm \\
\hline 283.7 & 3000 & 3000 & 0 & 276.5 & 1554 & 1500 & 54 \\
\hline 291.1 & 2839 & 2850 & -11 & 272.6 & 1387 & 1350 & 37 \\
\hline 290.2 & 2705 & 2700 & 5 & 269.7 & 1283 & 1200 & 83 \\
\hline 289.2 & 2564 & 2550 & 14 & 264.3 & 1129 & 1050 & 79 \\
\hline 287.7 & 2384 & 2400 & -16 & 255.1 & 937 & 900 & 37 \\
\hline 286.8 & 2286 & 2250 & 36 & 242.9 & 764 & 750 & 14 \\
\hline 286.1 & 2213 & 2100 & 113 & 220.4 & 571 & 600 & -29 \\
\hline 283.8 & 2007 & 1950 & 57 & 199.5 & 463 & 450 & 13 \\
\hline 281.4 & 1835 & 1800 & 35 & 152.1 & 325 & 300 & 25 \\
\hline 276.8 & 1570 & 1650 & -80 & 116.4 & 266 & 280 & -14 \\
\hline
\end{tabular}


With the increasing distance, the slope of the curve gradually increases; that is to say the greater of the variation of distance caused by the movement of the centroid location, the lower of the measurement resolution, which is a major cause of errors. ${ }^{[2]}$

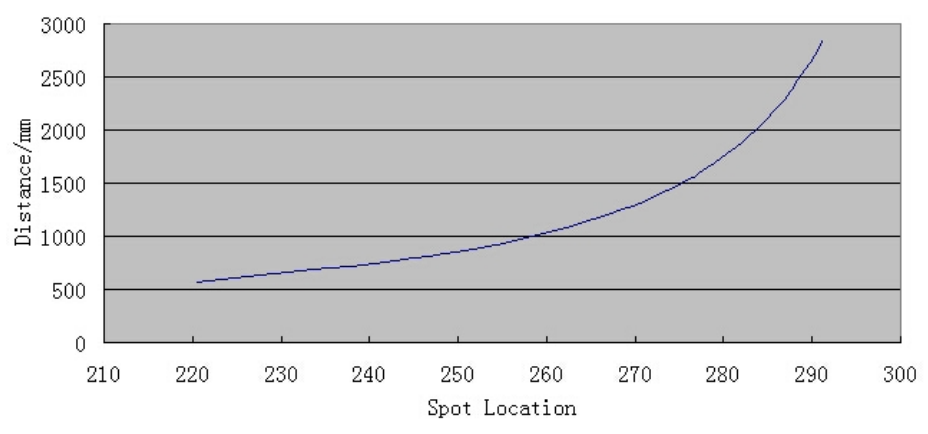

Figure 2

By the above analysis of experimental data, the reasons of errors are followed:

1. The image sensor resolution;

2. Artificial measurement error;

3. The installation of the distance measuring device errors, etc.

\section{Conclusions}

In this paper, the structure of the ranking system based on CMOS image sensor is simple. Through testing, the regional squared centroid method is easier and more accurate to calculate the spot centroid, and ranging error is in a reasonable range. The next task is to improve the accuracy for indoor positioning.

\section{References}

[1] Zhang Songmin, Wang Hong-lie, A new method of triangulation used for measuring medium and short distance[J]. Optical Technology,1999,9(5):49-53.

[2] Lin XiaoQian. Distance measuring using single - point laser triangulation system design based on CMOS [D], 2006.

[3] Liu Libo, Zhao Hui, Research on spot subdivided locating method in laser triangulation measurement [J]. Computer Measurement \& Control, 2008, 16(10).

[4] Su Yuwei, SUN Zhao, CHEN Yu. Based on image sensor and laser ranging method research [J]. Machinery \& Electronics, 2013(1). 